for the various water power developments of the Soviet Union.

The chapter on turbine regulation is adequate when dealing with possible layouts of equipment, and with the calculation of sizes of pressure receivers and the description of the interaction of governor components, but there is no mathematical treatment of the problem. It is possible that the various methods of analysis in this book would not be regarded as adequate by a mathematician, but the purpose of the book is clearly to teach design and it attempts to do so by the best available combination of theory and empirical results. Apart from a few slips, the translation is good. The value of the book would be much enhanced if only some of the many references quoted were to become available, for almost none of the formulae quoted are derived in the text.

E. GoldwaG

\section{VISCOUS FLUIDS}

\section{Low Reynolds Number Hydrodynamics with Special} Applications to Particulate Media

By John Happel and Howard Brenner. (Prentice-Hall International Series in the Physical and Chemical Engineering Sciences.) Pp. xiii +553 . (Englewood Cliffs, N.J., and London: Prentice-Hall Inc., 1965.) $120 s$.

The dimensionless quantity called the Reynolds number, defined as the product of typical values of the density, velocity and length scale, divided by the viscosity, is a measure of the importance of viscosity in a fluid flow. A Reynolds number small compared with unity implies that viscous effects are dominant and that the inertia or acceleration of the fluid is of secondary importance, the motion being governed by a balance between pressure forces and viscous stresses. Low Reynolds number hydrodynamics, or slow viscous flow, has been one of the less fashionable branches of fluid mechanics, lacking the glamour, possibly, of high speed aerodynamics and large scale geophysical phenomena. Yet, as the authors of this book point out in their introductory chapter, problems of slow viscous flow arise in many fields of science and technology from physics to medicine, including high speed aerodynamics. The field is full of fascinating and difficult problems, and interest has in fact been increasing in the last decade and a half. There is certainly a need for a comprehensive and authoritative text on the subject.

The greater part of this book, despite its length, is concerned with only one topic in the field-namely the problem of a particle moving through a fluid at rest and with the complete neglect of the acceleration terms, either in an unbounded fluid, in the presence of walls or in the presence of other particles. This topic is the one that lends itself most readily to mathematical analysis, and the authors have gone into great detail in describing the flow about spheres and ellipsoids and the forces on bodies of general shape. This material is likely to be valuable to students or to those wishing, for example, to know the drag on a sphere or ellipsoid moving in a pipe or channel. The non-mathematical reader may find the going hard, for although there is nothing intrinsically difficult in the analysis, the full panoply of the Gibbs vector notation is used and familiarity with curvilinear co-ordinates and eigenfunction expansions is assumed. There is some discussion of the important problem of particle motion in non-uniform flows, which would be the rule in many if not most applications, but it is somewhat limited. The intriguing effects of inertia on flow at low Reynolds number, which have become prominent in the last few years, are mentioned only in passing.

The last two chapters on flow relative to assemblages of particles (as, for example, in porous media) and the viscosity of suspensions give the impression of having been added so as to make the book of more practical interest.
They are not closely connected with the theme of the preceding six chapters. This part of the book does not contain much that is fresh or stimulating, but it may be considered a useful survey of these subjects.

In summary, this book provides a useful, detailed and connected account of the motion of small particles through uniform fluids at rest when the inertia is completely negligible. For accounts of lubrication theory, the effects of inertia, flows with free surfaces, motions of non-uniform fluids, and so on, the reader will have to look elsewhere.

P. G. SAFFMAN

\section{UNDERWATER ACOUSTICS}

\section{Applied Underwater Acoustics}

By D. G. Tucker and B. K. Gazey. (The Commonwealth and International Library of Science, Technology, Engineering and Liberal Studies: Physics Division.) Pp. xvi+ 244. (London and New York: Pergamon Press, Ltd., 1966.) $27 s .6 d$. net.

IN this text, the authors aim to provide an introduction to the fundamentals of acoustics as applied to underwater detection and communication. It is not possible in a small volume to cover in detail all aspects of the subject and here the accent is on transducer systems for which the authors rightly make no apology.

The first two chapters survey the general field of underwater acoustics from a modern point of view, and state the problems involved. An account of the relevant classical acoustic theory follows, leading to a general outline of sound propagation in the sea. In the last two chapters, which constitute nearly half the total text, the subject matter is discussed in more detail. A treatment of the theory and design of underwater transducers is followed by a lucid account of the theory of directional arrays and the synthesis of array patterns. The work concludes with a selection of problems and solutions.

Although intended primarily for students, this book should also be of value to those concerned with many aspects of underwater acoustics. It is up to date, has a good bibliography and is very reasonably pricod.

R. I. TAIT

\section{STRESS, TIME AND TEMPERATURE}

\section{Mathematical Theory of Creep and Creep Rupture} By K. G. Folke Odqvist. (Oxford Mathematical Monographs.) Pp. ix + 170. (Oxford: Clarendon Pross; London: Oxford University Press, 1966.) 48s. net.

THE word "fatigue" as a failure process in metallic materials is by now well known to laymen. The meaning of "creep", or the deformation of a material with time under specified conditions of stress and temperature, is almost equally universal. The original investigations of Andrade, between 1910 and 1914, established the classical relationship between strain and timo, but little attention was paid to this work by engineers until 1922, when Dickenson showed that short duration tensile tests at high temperature were completely misleading as a basis for the choice of materials for long service under high temperatures, even at low stress.

Since then, experimental work to improve the techniques of creep measurement has gone hand in hand with the use of engineering methods of stress analysis to prodict the lifetime of components. After 1945, the tremendous effort applied to dislocation theory in plasticity deflected. the main line of investigation towards a better understanding of the mechanisms of creep deformation and a proper appreciation of effects due to grain size, microstructure, and crack nucleation. The macro-picture cannot, as yet, be appreciated from this standpoint, as 